\title{
ITERATED SPINNING AND HOMOLOGY SPHERES
}

\author{
ALEXANDER I. SUCIU
}

\begin{abstract}
Given a closed $n$-manifold $M^{n}$ and a tuple of positive integers $P$, let $\sigma_{P} M$ be the $P$-spin of $M$. If $M^{n} \not S^{n}$ and $P \neq Q$ (as unordered tuples), it is shown that $\sigma_{P} M \not \sigma_{Q} M$ if either (1) $H_{*}\left(M^{n}\right) \not H_{*}\left(S^{n}\right),(2) \pi_{1} M$ finite, (3) $M$ aspherical, or (4) $n=3$. Applications to the homotopy classification of homology spheres and knot exteriors are given.
\end{abstract}

In this paper we study manifolds obtained by iterated $p$-spinning. The process of spinning was introduced by Artin [2] and generalized to $p$-spinning by Epstein [5] and Cappell [3]. As noticed by the latter, $p+q$-spinning is not in general the same as $p$-spinning followed by $q$-spinning. We expand on this remark of Cappell and show how to distinguish among the various iterated spins of a given manifold by means of their homology or the homology of their universal covers. As an application, we prove that successively spinning 3-manifolds in different ways produces manifolds with distinct homotopy types. Other applications concern homology spheres. For every $n \geq 5$, we construct homology $n$-spheres distinguished by their $r$ th homotopy groups $\left(2 \leq r \leq \frac{n-1}{2}\right)$, or by their first $k$-invariants. Finally, we discuss iterated spinning of knots.

\section{SPUN MANIFOLDS}

Let $M^{n}, n \geq 1$, be a closed, smooth $n$-manifold with basepoint *, and $p$ a positive integer. Let $D^{n}$ be a fixed embedded disk in $M^{n}$ and let $M_{0}^{n}=$ $M^{n} \backslash$ int $D^{n}$ be a punctured copy of $M$. The $p$-spin of $M$ is the closed, smooth $n+p$-manifold

$$
\sigma_{p} M=\partial\left(M_{0} \times D^{p+1}\right)=M_{0} \times S^{p} \cup_{S^{n-1} \times S^{p}} S^{n-1} \times D^{p+1} .
$$

In other words, $\sigma_{p} M$ is obtained from $M \times S^{p}$ by performing surgery on $* \times S^{p}$ with trivial framing. If $n \geq 3$, then $\sigma_{p} M$ has the same fundamental group as $M$ by Van Kampen's theorem. Another nice feature of $p$-spinning is that it respects connected sums.

Received by the editors April 5, 1988.

1980 Mathematics Subject Classification (1985 Revision). Primary 57R19; Secondary 55Q52, $57 \mathrm{Q} 45$.

Key words and phrases. $p$-spinning, homology sphere, homotopy type.

Partially supported by a grant from Northeastern University's Research and Scholarship Development Fund. 
Lemma 1.1 (Gordon [7]). $\sigma_{p}\left(M_{1} \# M_{2}\right) \cong \sigma_{p} M_{1} \# \sigma_{p} M_{2}$.

The process of $p$-spinning can of course be iterated. Given an $r$-tuple $P=$ $\left(p_{1}, \ldots, p_{r}\right), p_{i}>0$, we define the $P$-spin of $M$ to be

$$
\sigma_{P} M=\sigma_{p_{1}} \cdots \sigma_{p_{r}} M
$$

It is a closed, smooth manifold of dimension $n+|P|$, where $|P|=p_{1}+\cdots+p_{r}$. We also have $\pi_{1}\left(\sigma_{P} M\right) \cong \pi_{1} M$ (if $n \geq 3$ ) and $\sigma_{P}\left(M_{1} \# M_{2}\right) \cong \sigma_{P} M_{1} \# \sigma_{P} M_{2}$. The next lemma shows that $\sigma_{p} M$ depends only on the unordered tuple $P$.

Lemma 1.2. $\sigma_{p} \sigma_{q} M \cong \sigma_{q} \sigma_{p} M$.

Proof. Recall that $\sigma_{p} M$ is the result of surgery on $* \times S^{p}$ in $M \times S^{p}$. Therefore there is a cobordism $W_{p}(M)=M \times S^{p} \times I \cup h^{p+1}$ from $M \times S^{p}$ to $\sigma_{p} M$. Then $W_{p}(M) \times S^{q} \cong M \times S^{p} \times S^{q} \times I \cup h^{p+1} \cup h^{p+q+1}$ is a cobordism from $M \times S^{p} \times S^{q}$ to $\sigma_{p} M \times S^{q}$. Also $W_{q}\left(\sigma_{p} M\right)=\sigma_{p} M \times S^{q} \times I \cup h^{q+1}$ is a cobordism from $\sigma_{p} M \times S^{q}$ to $\sigma_{q} \sigma_{p} M$. Finally,

$$
\begin{aligned}
W_{q, p}(M) & =W_{p}(M) \times S^{q} \cup_{\sigma_{p} M \times S^{q}} W_{q}\left(\sigma_{p} M\right) \\
& =M \times S^{p} \times S^{q} \times I \cup h^{p+1} \cup h^{p+q+1} \cup h^{q+1}
\end{aligned}
$$

is a cobordism from $M \times S^{p} \times S^{q}$ to $\sigma_{q} \sigma_{p} M$. Now rearrange the handles to get

$$
W_{q, p}(M) \cong M \times S^{q} \times S^{p} \times I \cup h^{q+1} \cup h^{q+p+1} \cup h^{p+1}=W_{p, q}(M),
$$

a cobordism from $M \times S^{q} \times S^{p}$ to $\sigma_{p} \sigma_{q} M$. This proves the lemma.

If $M$ is a manifold admitting a simple geometric description it is often not too difficult to identify $\sigma_{p} M$. For example, $\sigma_{p} S^{n}$ is $S^{n+p}$. We leave as an exercise for the reader to show $\sigma_{p}\left(\mathbb{R P}^{n}\right)=S^{n-1} \times_{\mathbb{Z}_{2}} S^{p+1}$ (where the generator of $\mathbb{Z}_{2}$ acts by (antipodal, reflection)), $\sigma_{p}\left(\mathbb{C P}^{n}\right)=\mathbb{C P}^{n-1} \times S^{p+2}$, and $\sigma_{p}\left(\mathbb{H} \mathbb{P}^{n}\right)=\mathbb{H P}^{n-1} \times S^{p+4}$. What we really need is to identify the $P$-spins of product of spheres.

Lemma 1.3. (i) $\sigma_{p}\left(S^{m} \times S^{n}\right) \cong S^{m+p} \times S^{n} \# S^{m} \times S^{n+p}$.

(ii) $\sigma_{P}\left(S^{m} \times S^{n}\right) \cong \#_{R \subseteq P} S^{m+|R|} \times S^{n+|P|-|R|}$.

Proof. (i) By definition, $\sigma_{p}\left(S^{m} \times S^{n}\right)=\partial W$, where $W=\left(S^{m} \times S^{n}\right)_{0} \times D^{p+1}$. As $\left(S^{m} \times S^{n}\right)_{0} \cong D^{m+n} \cup h^{m} \cup h^{n}$, we have $W \cong D^{m+n+p+1} \cup h^{m} \cup h^{n}$. The attaching spheres for the handles of $W$ form a link $S^{m-1} \cup S^{n-1} \subset S^{m+n+p}$ of codimension $p+2>2$. By general position this is the unlink. Thus

$$
\begin{aligned}
W & \cong\left(D^{m+n+p+1} \cup h^{m}\right) \curvearrowleft\left(D^{m+n+p+1} \cup h^{n}\right) \\
& \cong S^{m} \times D^{n+p+1} \curvearrowleft S^{n} \times D^{m+p+1} .
\end{aligned}
$$

This implies $\partial W \cong S^{m} \times S^{n+p} \# S^{n} \times S^{m+p}$. 
(ii) Follows from (i) and Lemma 1.1. The connected sum is taken over all subsets $R$ of $P$, including $R=P$ and $R=\varnothing$ (where $|\varnothing|=0$ ).

In particular, we have a complete description of spins of 2-manifolds:

$$
\sigma_{p}\left(S^{2}\right)=S^{p+2}, \quad \sigma_{p}\left(\#_{1}^{g} S^{1} \times S^{1}\right)=\#_{1}^{2 g} S^{p+1} \times S^{1}, \quad \sigma_{p}\left(\#_{1}^{h} \mathbb{R} \mathbb{P}^{2}\right)=\#_{1}^{h} S^{p+1} \widetilde{\times} S^{1} .
$$

Spun 3-manifolds were studied in [13], where it is shown that if $M^{3}$ is oriented and geometric, then $\pi_{1} M$ determines the oriented homotopy type of $\sigma_{1} M$.

We are now in position to describe the universal cover of the $P$-spin of $M^{n}, n \geq 3$, in terms of the $P$-spin of the universal cover of $M^{n}$. This will generalize the description in Plotnick [10], done there for $n=3$ and $p=1$. First consider $\widetilde{\sigma_{p} M}$. Let $\pi=\pi_{1} M \cong \pi_{1}\left(\sigma_{p} M\right)$. Then $\widetilde{\sigma_{p} M}$ is obtained by taking the cover $\widetilde{M} \times S^{p}$ of $M \times S^{p}$ and performing (equivariant) surgery on the $|\pi|$ lifts of $* \times S^{p}$. That is,

$$
\widetilde{\sigma_{p} M}=\widetilde{M}_{0} \times S^{p} \bigcup_{\pi} S^{n-1} \times S^{p} \coprod_{\pi} S^{n-1} \times D^{p+1}
$$

The first surgery yields $\sigma_{p} \widetilde{M}$. The other $|\pi|-1$ surgeries are done on nullhomotopic $S^{p}$ 's, so they add copies of $S^{n-1} \times S^{p+1}$. Therefore

$$
\widetilde{\sigma_{p} M} \cong \sigma_{p} \widetilde{M} \# \#_{1}^{|\pi|-1} S^{n-1} \times S^{p+1}
$$

Two particular cases are worth mentioning:

$$
\begin{aligned}
& \text { If } M=S^{n} / \pi, \text { then } \widetilde{\sigma_{p} M} \cong \#_{1}^{|\pi|-1} S^{n-1} \times S^{p+1} ; \\
& \text { If } M=\mathbb{R}^{n} / \pi, \text { then } \widetilde{\sigma_{p} M} \cong S^{n-1} \times \mathbb{R}^{p+1} \# \#_{1}^{\infty} S^{n-1} \times S^{p+1} .
\end{aligned}
$$

We now generalize to iterated spins.

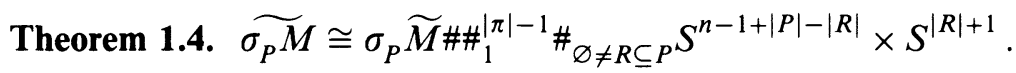

Proof. We use the above formula, Lemmas 1.1 and 1.3, and induction on length $(P)$. If $P=\left(p, P^{\prime}\right)$ then

$$
\begin{aligned}
\widetilde{\sigma_{P} M}= & \sigma_{p}\left(\widetilde{\sigma_{P^{\prime}}} M\right) \cong \sigma_{p}\left(\widetilde{\sigma_{P^{\prime}} M}\right) \# \#_{1}^{|\pi|-1} S^{n-1+\left|P^{\prime}\right|} \times S^{p+1} \\
\cong & \sigma_{p}\left(\sigma_{P^{\prime}} \widetilde{M} \# \# \#_{1}^{|\pi|-1} \#_{\varnothing \neq R^{\prime} \subseteq P^{\prime}} S^{n-1+\left|P^{\prime}\right|-\left|R^{\prime}\right|} \times S^{\left|R^{\prime}\right|+1}\right) \# \#_{1}^{|\pi|-1} S^{n-1+\left|P^{\prime}\right|} \times S^{p+1} \\
\cong & \sigma_{p}\left(\sigma_{P^{\prime}} \widetilde{M}\right) \# \#_{1}^{|\pi|-1} \# \varnothing \neq R^{\prime} \subseteq P^{\prime}\left(S^{n-1+|P|-\left|R^{\prime}\right|} \times S^{\left|R^{\prime}\right|+1}\right. \\
& \left.\# S^{n-1+|P|-\left|R^{\prime}\right|-p} \times S^{\left|R^{\prime}\right|+p+1}\right) \# \#_{1}^{|\pi|-1} S^{n-1+\left|P^{\prime}\right|} \times S^{p+1} \\
= & \sigma_{P} \widetilde{M} \# \#_{1}^{|\pi|-1} \#_{\varnothing \neq R \subseteq P} S^{n-1+|P|-|R|} \times S^{|R|+1} .
\end{aligned}
$$

\section{Homology of $\sigma_{P} M$}

In this section we compute the homology groups of $\sigma_{P} M$ and show how quite often they can be used to distinguish between $\sigma_{P} M$ and $\sigma_{Q} M$, if $P \neq Q$. Of course, if $|P| \neq|Q|$, then $\operatorname{dim} \sigma_{P} M \neq \operatorname{dim} \sigma_{Q} M$ and there is nothing to prove. 
So from now on we will implicitly assume $|P|=|Q|$ whenever we compare $\sigma_{P} M$ to $\sigma_{Q} M$.

Lemma 2.1. $H_{i}\left(\sigma_{p} M\right) \cong H_{i}\left(M_{0}\right) \oplus \widetilde{H}_{i-p}(M)$.

Proof. The Mayer-Vietoris sequence for the decomposition $\sigma_{p} M=M_{0} \times S^{p}$ $\cup_{S^{n-1} \times S^{p}} S^{n-1} \times D^{p+1}$ reduces to the exact sequences

$$
\begin{aligned}
& 0 \rightarrow H_{i}\left(M_{0} \times S^{p}\right) \rightarrow H_{i}\left(\sigma_{p} M\right) \rightarrow 0, \quad i \neq p, p+n-1, p+n ; \\
& 0 \rightarrow H_{p}\left(S^{p}\right) \rightarrow H_{p}\left(M_{0} \times S^{p}\right) \rightarrow H_{p}\left(\sigma_{p} M\right) \rightarrow 0 ; \\
& \begin{aligned}
0 \rightarrow H_{p+n}\left(\sigma_{p} M\right) \rightarrow H_{p+n-1}\left(S^{n-1} \times S^{p}\right) \rightarrow H_{p+n-1}\left(M_{0} \times S^{p}\right) \\
\qquad H_{p+n-1}\left(\sigma_{p} M\right) \rightarrow 0 .
\end{aligned}
\end{aligned}
$$

The result follows from the Künneth formula.

Theorem 2.2. $H_{i}\left(\sigma_{P} M\right) \cong H_{i}\left(M_{0}\right) \oplus \bigoplus_{\varnothing \neq R \subset P} \widetilde{H}_{i-|R|}\left(M_{0}\right) \oplus \widetilde{H}_{i-|P|}(M)$.

Proof. It suffices to prove that $\tilde{H}_{i}\left(\sigma_{P} M_{0}\right) \cong \bigoplus_{R \subseteq P} \widetilde{H}_{i-|R|}\left(M_{0}\right)$. This is done by induction on length $(P)$. For $P=(p)$, this is immediate from Lemma 2.1 . For $P=\left(p, P^{\prime}\right)$ we have

$$
\begin{aligned}
\widetilde{H}_{i}\left(\sigma_{P} M_{0}\right) & =\widetilde{H}_{i}\left(\sigma_{p}\left(\sigma_{P^{\prime}} M\right)_{0}\right) \\
& \cong \widetilde{H}_{i}\left(\sigma_{P^{\prime}} M_{0}\right) \oplus \widetilde{H}_{i-p}\left(\sigma_{P^{\prime}} M_{0}\right) \\
& \cong \bigoplus_{R^{\prime} \subseteq P^{\prime}} \widetilde{H}_{i-\left|R^{\prime}\right|}\left(M_{0}\right) \oplus \bigoplus_{R^{\prime} \subseteq P^{\prime}} \widetilde{H}_{i-p-\left|R^{\prime}\right|}\left(M_{0}\right) \\
& =\bigoplus_{R \subseteq P} \widetilde{H}_{i-|R|}\left(M_{0}\right) .
\end{aligned}
$$

In particular, if $M^{n}$ is a homology $n$-sphere, that is if $H_{*}\left(M^{n}\right) \cong H_{*}\left(S^{n}\right)$, then $\sigma_{P} M$ is a homology $n+|P|$-sphere. This means that we cannot distinguish homologically among the various iterated spins of a given homology sphere (unless they have different dimensions). For other manifolds though, we have the following:

Theorem 2.3. If $H_{*}\left(M^{n}\right) \not H_{*}\left(S^{n}\right)$ and $P \neq Q$ (as unordered tuples), then $H_{*}\left(\sigma_{P} M\right) \not H_{*}\left(\sigma_{Q} M\right)$.

Proof. By assumption we have $\widetilde{H}_{*}\left(M_{0}^{n}\right) \not \widetilde{H}_{*}\left(D^{n}\right)$, so let $i_{0}$ be the smallest integer such that $\tilde{H}_{i_{0}}\left(M_{0}^{n}\right) \neq 0$. Arrange $P$ and $Q$ in ascending order and assume $P \prec Q$. Let $j$ be the smallest integer such that $p_{j}<q_{j}$. Then write $P=\left(P^{\prime}, P^{\prime \prime}\right), Q=\left(P^{\prime}, Q^{\prime \prime}\right)$, where $P^{\prime}=\left(p_{1} \leq \cdots \leq p_{j-1}\right)$ and $P^{\prime \prime}=\left(p_{j}=\right.$ $\cdots=p_{j+k-1}<p_{j+k} \leq \cdots \leq p_{r}$ ), for some $k \geq 1$. (If $j=1, P=P^{\prime \prime}, Q=Q^{\prime \prime}$.) For $i<p_{j}+i_{0}$, one can check that $H_{i}\left(\sigma_{P} M\right) \cong H_{i}\left(\sigma_{Q} M\right)$. For $i=p_{j}+i_{0}$ 
though, we have

$$
\begin{aligned}
H_{p_{j}+i_{0}}\left(\sigma_{P} M\right) \cong & H_{p_{j}+i_{0}}\left(\sigma_{P^{\prime}} M_{0}\right) \\
& \oplus\left(\bigoplus_{\varnothing \neq R^{\prime \prime} \subset P^{\prime \prime}} \widetilde{H}_{p_{j}+i_{0}-\left|R^{\prime \prime}\right|}\left(\sigma_{P^{\prime}} M_{0}\right) \oplus \widetilde{H}_{p_{j}+i_{0}-\left|P^{\prime \prime}\right|}\left(\sigma_{P^{\prime}} M\right)\right),
\end{aligned}
$$

where $\sigma_{P^{\prime}} M=M$ in case $j=1$. The assumption on $i_{0}$ implies that the only nonzero terms in the above parentheses are those corresponding to $R^{\prime \prime}=$ $\left(p_{l}\right), j \leq l \leq j+k-1$, which contribute

$$
\widetilde{H}_{p_{j}+i_{0}-\left|R^{\prime \prime}\right|}\left(\sigma_{P^{\prime}} M_{0}\right) \cong \widetilde{H}_{i_{0}}\left(\sigma_{P^{\prime}} M_{0}\right) \cong \bigoplus_{R^{\prime} \subset P^{\prime}} \widetilde{H}_{i_{0}-\left|R^{\prime}\right|}\left(M_{0}\right) \cong \widetilde{H}_{i_{0}}\left(M_{0}\right) \text {. }
$$

Thus

$$
H_{p_{j}+i_{0}}\left(\sigma_{P} M\right) \cong H_{p_{j}+i_{0}}\left(\sigma_{P^{\prime}} M\right) \oplus\left(\widetilde{H}_{i_{0}}\left(M_{0}\right)\right)^{k}
$$

Similarly

$$
H_{p_{j}+i_{0}}\left(\sigma_{Q} M\right) \cong H_{p_{j}+i_{0}}\left(\sigma_{P^{\prime}} M\right)
$$

As all groups in question are finitely generated abelian, we conclude that

$$
H_{p_{j}+i_{0}}\left(\sigma_{P} M\right) \not H_{p_{j}+i_{0}}\left(\sigma_{Q} M\right) \text {. }
$$

\section{HoMology of $\widetilde{\sigma_{P} M}$}

We now show how to distinguish among the iterated spins of $M^{n}, n \geq 3$, by means of the homology of their universal covers. We start by describing the homology groups of $\widetilde{\sigma_{p} M}$, viewed as $\mathbb{Z} \pi$-modules, where $\pi=\pi_{1} M \cong$ $\pi_{1}\left(\sigma_{p} M\right)$. Let $I \pi$ be the augmentation ideal $\operatorname{ker}(\mathbb{Z} \pi \stackrel{\varepsilon}{\rightarrow} \mathbb{Z})$ and $\delta($,$) the$ Kronecker delta. To keep the notation compact we adopt the convention $A^{0}=$ 0 , for a module $A$.

Lemma 3.1. $H_{i}\left(\widetilde{\sigma_{p} M}\right) \cong H_{i}\left(\widetilde{M_{0}}\right) \oplus \widetilde{H}_{i-p}(\widetilde{M}) \oplus(I \pi)^{\delta(i-1, p)}$.

Proof. The Mayer-Vietoris sequence for the decomposition

$$
\widetilde{\sigma_{p} M}=\widetilde{M}_{0} \times S^{p} \bigcup_{\pi} S^{n-1} \times S^{p} \coprod_{\pi} S^{n-1} \times D^{p+1}
$$

reduces to the exact sequences

$$
\begin{aligned}
& 0 \rightarrow H_{i}\left(\widetilde{M_{0}} \times S^{p}\right) \rightarrow H_{i}\left(\widetilde{\sigma_{p} M}\right) \rightarrow 0, \quad i \neq p, p+1, p+n-1, p+n \\
& 0 \rightarrow H_{p+1}\left(\widetilde{M_{0}}\right) \rightarrow H_{p+1}\left(\widetilde{\sigma_{p} M}\right) \rightarrow \bigoplus_{\pi} H_{p}\left(S^{p}\right) \underset{(0, \varepsilon)}{\rightarrow} H_{p}\left(\widetilde{M_{0}}\right) \oplus H_{p}\left(S^{p}\right) \rightarrow H_{p}\left(\widetilde{\sigma_{p} M}\right) \rightarrow 0 ; \\
& \mathbb{Z} \pi \quad \mathbb{Z} \\
& 0 \rightarrow H_{p+n}\left(\widetilde{\sigma_{p} M}\right) \rightarrow \bigoplus_{\pi} H_{p+n-1}\left(S^{n-1} \times S^{p}\right) \rightarrow H_{p+n-1}\left(\widetilde{M_{0}} \times S^{p}\right) \rightarrow H_{p+n-1}\left(\widetilde{\sigma_{p} M}\right) \rightarrow 0 \\
& \bigoplus_{\pi} H_{n-1}^{\mathbb{R}}\left(S^{n-1}\right) \quad H_{n-1}\left(\widetilde{M}_{0}\right)
\end{aligned}
$$


Thus

$$
H_{i}\left(\widetilde{\sigma_{p} M}\right) \cong \begin{cases}H_{i}\left(\widetilde{M_{0}}\right), & i<p+1, \\ H_{i}\left(\widetilde{M_{0}}\right) \oplus I \pi, & i=p+1, \\ H_{i}\left(\widetilde{M_{0}}\right) \oplus H_{i-p}\left(\widetilde{M_{0}}\right), & p+1<i<p+n-1 \\ H_{i}(\widetilde{M}), & i \geq p+n-1 .\end{cases}
$$

We are now ready to describe the homology of $\widetilde{\sigma_{P} M}$. For $P$ a tuple we will use a generalized Kronecker delta given by $\Delta(i, P)=\sum_{\varnothing \neq R \subseteq P} \delta(i,|R|)$.

\section{Theorem 3.2.}

$$
H_{i}\left(\widetilde{\sigma_{P} M}\right) \cong H_{i}\left(\widetilde{M_{0}}\right) \oplus \bigoplus_{\varnothing \neq R \subset P} \widetilde{H}_{i-|R|}\left(\widetilde{M_{0}}\right) \oplus \widetilde{H}_{i-|P|}(\widetilde{M}) \oplus(I \pi)^{\Delta(i-1, P)} .
$$

Proof. It suffices to prove that $\widetilde{H}_{i}\left(\sigma_{P} M_{0}\right) \cong \bigoplus_{R \subseteq P} \widetilde{H}_{i-|R|}\left(\widetilde{M_{0}}\right) \oplus(I \pi)^{\Delta(i-1, P)}$. Again this is done by induction on length $(\mathrm{P})$. If $P=\left(p, P^{\prime}\right)$, then

$$
\begin{aligned}
& \widetilde{H}_{i}\left(\widetilde{\sigma_{P} M_{0}}\right)=\widetilde{H}_{i}\left(\widetilde{\sigma_{p}}\left(\widetilde{\sigma_{P^{\prime}} M}\right)_{0}\right) \\
& \cong \widetilde{H}_{i}\left(\widetilde{\sigma_{P^{\prime}} M_{0}}\right) \oplus \widetilde{H}_{i-p}\left(\widetilde{\sigma_{P^{\prime}} M_{0}}\right) \oplus(I \pi)^{\delta(i-1, p)} \\
& \cong\left(\bigoplus_{R^{\prime} \subseteq P^{\prime}} \widetilde{H}_{i-|R|}\left(\widetilde{M_{0}}\right) \oplus(I \pi)^{\Delta\left(i-1, P^{\prime}\right)}\right) \\
& \oplus\left(\bigoplus_{R^{\prime} \subseteq P^{\prime}} \widetilde{H}_{i-p-\left|R^{\prime}\right|}\left(\widetilde{M_{0}}\right) \oplus(I \pi)^{\Delta\left(i-p-1, P^{\prime}\right)}\right) \oplus(I \pi)^{\delta(i-1, p)} \\
& =\bigoplus_{R \subseteq P} \widetilde{H}_{i-|R|}\left(\widetilde{M_{0}}\right) \oplus(I \pi)^{\Delta(i-1, P)} .
\end{aligned}
$$

We now would like to distinguish between $H_{*}\left(\widetilde{\sigma_{P} M}\right)$ and $H_{*}\left(\widetilde{\sigma_{Q} M}\right)$, provided $P \neq Q$. For that we adopt the notation from the proof of Theorem 2.3. $P=\left(P^{\prime}, P^{\prime \prime}\right)$, with $P^{\prime}=\left(p_{1} \leq \cdots \leq p_{j-1}\right), P^{\prime \prime}=\left(p_{j}=\cdots=p_{j+k-1}<p_{j+k} \leq\right.$ $\left.\cdots \leq p_{r}\right)$ and $Q=\left(P^{\prime}, Q^{\prime \prime}\right)$, with $Q^{\prime \prime}=\left(q_{j} \leq \cdots \leq q_{s}\right)$, where $p_{j}<q_{j}$ and $k \geq 1$. For $i \leq p_{j}$, we have $H_{i}\left(\widetilde{\sigma_{P} M}\right) \cong H_{i}\left(\widetilde{\sigma_{Q} M}\right)$, as can be seen by direct computation or by using Lemma 5.1 below. For $i=p_{j}+1$ however, we have

Lemma 3.3. $H_{p_{j}+1}\left(\widetilde{\sigma_{P} M}\right) \cong H_{p_{j}+1}\left(\widetilde{\sigma_{Q} M}\right) \oplus(I \pi)^{k}$. 
Proof. We compute

$$
\begin{aligned}
H_{p_{j}+1} & \left(\widetilde{\sigma_{P} M}\right)=H_{p_{j}+1}\left(\sigma_{P^{\prime \prime}}\left(\sigma_{P^{\prime}} M\right)\right) \\
\cong & H_{p_{j}+1}\left(\sigma_{P^{\prime}} M_{0}\right) \oplus \bigoplus_{\varnothing \neq R^{\prime \prime} \subset P^{\prime \prime}} \widetilde{H}_{p_{j}+1-\left|R^{\prime \prime}\right|}\left(\sigma_{P^{\prime}} M_{0}\right) \\
& \oplus \widetilde{H}_{p_{j}+1-\left|P^{\prime \prime}\right|}\left(\widetilde{\sigma_{P^{\prime}} M}\right) \oplus(I \pi)^{\Delta\left(p_{j}, P^{\prime \prime}\right)} \\
\cong & H_{p_{j}+1}\left(\widetilde{\sigma_{P^{\prime}} M_{0}}\right) \oplus \bigoplus_{\varnothing \neq R^{\prime \prime} \subset P^{\prime \prime}} \bigoplus_{R^{\prime} \subseteq P^{\prime}} \widetilde{H}_{p_{j}+1-\left|R^{\prime \prime}\right|-\left|R^{\prime}\right|}\left(\widetilde{M_{0}}\right) \\
& \oplus \bigoplus_{R^{\prime} \subset P^{\prime}} \widetilde{H}_{p_{j}+1-\left|P^{\prime \prime}\right|-\left|R^{\prime}\right|}\left(\widetilde{M_{0}}\right) \\
& \oplus \widetilde{H}_{p_{j}+1-\left|P^{\prime \prime}\right|-\left|P^{\prime}\right|}(\widetilde{M}) \oplus \bigoplus_{\varnothing \neq R^{\prime \prime} \subseteq P^{\prime \prime}}(I \pi)^{\Delta\left(p_{j}-\left|R^{\prime \prime}\right|, P^{\prime}\right)} \oplus(I \pi)^{\Delta\left(p_{j}, P^{\prime \prime}\right)} .
\end{aligned}
$$

For $\varnothing \neq R^{\prime \prime} \subseteq P^{\prime \prime}$ and $R^{\prime} \subseteq P^{\prime}$ we have $p_{j}-\left|R^{\prime \prime}\right| \leq 0$, so $p_{j}+1-\left|R^{\prime \prime}\right|-\left|R^{\prime}\right| \leq 1$. This implies $\widetilde{H}_{p_{j}+1-\left|R^{\prime \prime}\right|-\left|R^{\prime}\right|}\left(\widetilde{M_{0}}\right)=\widetilde{H}_{p_{j}+1-\left|P^{\prime \prime}\right|-\left|P^{\prime}\right|}(\widetilde{M})=0$. Also

$$
\Delta\left(p_{j}, P^{\prime \prime}\right)=\sum_{\varnothing \neq R^{\prime \prime} \subseteq P^{\prime \prime}} \delta\left(p_{j},\left|R^{\prime \prime}\right|\right)=k
$$

and

$$
\Delta\left(p_{j}-\left|R^{\prime \prime}\right|, P^{\prime}\right)=\sum_{\varnothing \neq R^{\prime} \subseteq P^{\prime}} \delta\left(p_{j}-\left|R^{\prime \prime}\right|,\left|R^{\prime}\right|\right)=0,
$$

as $p_{j}-\left|R^{\prime \prime}\right| \leq 0$ and $\left|R^{\prime}\right|>0$. Therefore

$$
H_{p_{j}+1}\left(\widetilde{\sigma_{P} M}\right) \cong H_{p_{j}+1}\left(\widetilde{\sigma_{P^{\prime}} M_{0}}\right) \oplus(I \pi)^{k}
$$

Similarly,

finishing the proof.

$$
H_{p_{j}+1}\left(\widetilde{\sigma_{Q^{M}}}\right) \cong H_{p_{j}+1}\left(\widetilde{\sigma_{P^{\prime}} M_{0}}\right)
$$

In order to be able to distinguish among the $\mathbb{Z} \pi$-modules in Lemma 3.3 we need a cancellation theorem. Namely, given $\pi$ a finitely generated group, and $A, B$ finitely-generated $\mathbb{Z} \pi$-modules, we want to conclude that $A \oplus B \cong A \Rightarrow$ $B=0$. Such a general result holds if $A$ is projective. For then we have $(\mathbb{Z} \pi)^{l} \oplus B \cong(\mathbb{Z} \pi)^{l}$, which implies $B=0$ by a theorem of Kaplansky [9]. But in our situation $A=H_{p_{j}+1}\left(\widetilde{\sigma_{P} M}\right)$ need not be projective, so we will settle for two particular cases.

Corollary 3.4. If $\pi_{1} M$ is a finite, nontrivial group and $P \neq Q$, then $H_{*}\left(\widetilde{\sigma_{P} M}\right) \neq$ $H_{*}\left(\widetilde{\sigma_{Q} M}\right)$ as abelian groups.

Proof. We have

$$
\begin{aligned}
\operatorname{rank}\left(H_{p_{j}+1}\left(\widetilde{\sigma_{P} M}\right)\right) & =\operatorname{rank}\left(H_{p_{j}+1}\left(\widetilde{\sigma_{Q} M}\right)\right)+k(|\pi|-1) \\
& >\operatorname{rank}\left(H_{p_{j}+1}\left(\widetilde{\sigma_{Q} M}\right)\right)
\end{aligned}
$$


Corollary 3.5. If $M$ is aspherical and $P \neq Q$, then $H_{*}\left(\widetilde{\sigma_{P} M}\right) \neq H_{*}\left(\widetilde{\sigma_{Q} M}\right)$ as $\mathbb{Z} \pi$-modules.

Proof. Since $\widetilde{M}$ is contractible, $\widetilde{H}_{i}\left(\widetilde{M_{0}}\right)=0$ for $i \neq n-1$ and $\widetilde{H}_{n-1}\left(\widetilde{M_{0}}\right) \cong$ $\mathbb{Z} \pi$. Thus:

$$
\begin{aligned}
H_{p_{j}+1}\left(\sigma_{P^{\prime}} M_{0}\right) & \cong \bigoplus_{R^{\prime} \subseteq P^{\prime}} \widetilde{H}_{p_{j}+1-\left|R^{\prime}\right|}\left(\widetilde{M}_{0}\right) \oplus(I \pi)^{\Delta\left(p_{j}, P^{\prime}\right)} \\
& \cong(\mathbb{Z} \pi)^{\delta\left(p_{j}-n+2,0\right)+\Delta\left(p_{j}-n+2, P^{\prime}\right)} \oplus(I \pi)^{\Delta\left(p_{j}, P^{\prime}\right)} .
\end{aligned}
$$

As $\pi$ is a Poincaré-duality group of dimension $n>1, H^{1}(\pi, \mathbb{Z} \pi)=0$. The cohomology exact sequence associated to the coefficient sequence $0 \rightarrow I \pi \rightarrow$ $\mathbb{Z} \pi \stackrel{\varepsilon}{\rightarrow} \mathbb{Z} \rightarrow 0$ yields $H^{1}(\pi, I \pi) \cong H^{0}(\pi, \mathbb{Z}) \cong \mathbb{Z}$. Therefore

$$
\begin{aligned}
\operatorname{rank} H^{1}\left(\pi, H_{p_{j}+1}\left(\widetilde{\sigma_{P} M}\right)\right) & =\operatorname{rank} H^{1}\left(\pi, H_{p_{j}+1}\left(\widetilde{\sigma_{Q} M}\right)\right)+k \\
& >\operatorname{rank} H^{1}\left(\pi, H_{p_{j}+1}\left(\widetilde{\sigma_{Q} M}\right)\right) .
\end{aligned}
$$

\section{HOMOTOPY TYPE OF ITERATED SPINS}

In view of the above results it seems reasonable to make the following:

Conjecture. If $M^{n} \not S^{n}$ and $P \neq Q$, then $\sigma_{P} M \not \sigma_{Q} M$.

It follows from 2.3, 3.4 and 3.5 that the conjecture is true if either

(1) $M^{n}$ is not a homology sphere, or

(2) $M^{n}$ is a homology sphere with $\pi_{1} M$ finite, or

(3) $M^{n}$ is an aspherical homology sphere.

We showed in $\S 3$ how to reduce this problem to a cancellation theorem for $\mathbb{Z} \pi$ modules. If $n=3$ we can bypass this seemingly difficult algebraic question by using some geometric arguments.

Theorem 4.1. If $M^{3} \not S^{3}$ and $P \neq Q$, then $\sigma_{P} M \not \sigma_{Q} M$.

Proof. Suppose $\sigma_{P} M \simeq \sigma_{Q} M$. By the above, we only have to consider the case when $M$ is a homology 3-sphere. In particular, $M$ is orientable. Then $M$ admits a prime factorization $M=M_{1} \# \ldots \# M_{l}$ (see [8]). The summands $M_{i}$ are homology 3-spheres, with $\widetilde{M}_{i} \simeq S^{3}$ or $\widetilde{M}_{i} \simeq \mathbb{R}^{3}$. We may assume $\pi_{1} M_{i} \neq 1$, as homotopy sphere summands do not affect the homotopy type of $M$ or that of its spins. We may also assume $l \geq 2$, for otherwise $M=M_{1}$ is of type (2) or (3) and $\sigma_{P} M \not \sigma_{Q} M$.

Now let $p$ be a nonnegative integer so that $|P|+p=|Q|+p=4 m$, for some $m>0$, and set $\bar{P}=(P, p), \bar{Q}=(Q, p)$. Clearly $\bar{P} \neq \bar{Q}$ and $\sigma_{\bar{P}} M \simeq \sigma_{\bar{Q}} M$. By Lemma 1.1 there exists a homotopy equivalence

$$
\sigma_{\bar{P}}\left(M_{1}\right) \# \sigma_{\bar{P}}\left(M_{2} \# \cdots \# M_{l}\right) \simeq \sigma_{\bar{Q}}\left(M_{1}\right) \# \sigma_{\bar{Q}}\left(M_{2} \# \cdots \# M_{l}\right)
$$

between closed, orientable manifolds of dimension $4 m+3 \geq 7$. By Cappell's Splitting Theorem [4, Theorem 3] the homotopy equivalence is splittable, and 
we get either

$$
\begin{aligned}
& \sigma_{\bar{P}}\left(M_{1}\right) \simeq \sigma_{\bar{Q}}\left(M_{1}\right), \text { or } \\
& \sigma_{\bar{P}}\left(M_{1}\right) \simeq \sigma_{\bar{Q}}\left(M_{2} \# \cdots \# M_{l}\right) .
\end{aligned}
$$

In the first case we derive a contradiction as before. In the second case, we must have $l=2$, for otherwise $\pi_{1} M_{1}$ is a nontrivial free product. Hence $\sigma_{\bar{P}} M_{1} \simeq$ $\sigma_{\bar{Q}} M_{2}$. This implies $\pi_{1} M_{1} \cong \pi_{1} M_{2}$ and thus $\widetilde{M}_{1} \simeq \widetilde{M}_{2}$. It follows from the proof of Theorem 3.5 that $\left.H_{*}\left(\widetilde{\sigma_{\bar{P}} M_{1}}\right) \not H_{*}\left(\widetilde{\sigma_{\bar{Q}}}\right)_{2}\right)$-a contradiction.

The above proof may be adapted to show that the Conjecture holds for connected sums of manifolds of type (1), (2) or (3). But of course not all manifolds of dimension $>3$ can be so decomposed. For example, let $\Sigma^{3}$ be the Poincaré sphere and form $S^{1} \times \Sigma^{3} \# S^{1} \times \Sigma^{3}$, with fundamental group

$$
\begin{aligned}
& \left(t, a, b \mid a^{3}=b^{5}=(a b)^{2},[t, a]=[t, b]=1\right) \\
& \quad *\left(u, c, d \mid c^{3}=d^{5}=(c d)^{2},[u, c]=[u, d]=1\right) .
\end{aligned}
$$

Then perform surgery on the curves $t(c d)^{2}$ and $u(a b)^{2}$. The resulting manifold is a homology 4-sphere $M^{4}$ with

$$
\pi_{1} M^{4}=\mathbb{Z}_{2} \times \pi_{1} \Sigma *_{\mathbb{Z}_{2} \times \mathbb{Z}_{2}} \mathbb{Z}_{2} \times \pi_{1} \Sigma .
$$

This group is not a free product, so $M \not M_{1} \# M_{2}$ (unless $M_{1}$ or $M_{2}$ is $\simeq S^{4}$ ). Moreover, the center $\mathbb{Z}_{2} \times \mathbb{Z}_{2}$ is not square-root closed in $\pi_{1} M$, so none of Cappell's splitting theorems apply.

\section{HOMOLOGY SPHERES}

We now use the techniques of the previous sections to produce examples of homology spheres distinguished by certain homotopy type invariants. First recall a definition from [15]. Two connected CW-complexes $K_{1}$ and $K_{2}$ have the same $p$-type ${ }^{1}$ if there is a cellular map $f: K_{1}^{(p+1)} \rightarrow K_{2}^{(p+1)}$ with $\left.f\right|_{K_{1}^{(p)}}$ a homotopy equivalence. If $K_{1}$ and $K_{2}$ are of the same $p$-type, they have isomorphic homotopy groups $\pi_{i}(i=1, \ldots, p)$ and $k$-invariants $k_{i}(i=1, \ldots, p-1)$. Now let $P=\left(p_{1}, \ldots, p_{r}\right), Q=\left(q_{1}, \ldots, q_{s}\right)$.

Lemma 5.1. $\sigma_{P} M$ and $\sigma_{Q} M$ have the same p-type, for

$$
p=\min \left(p_{1}, \ldots, p_{r}, q_{1}, \ldots, q_{s}\right) \text {. }
$$

Proof. Recall $\sigma_{p} M=M_{0} \times S^{p} \cup S^{n-1} \times D^{p+1}$. It follows that $\sigma_{p} M$ admits a CWdecomposition with $\left(\sigma_{p} M\right)^{(p)} \simeq\left(M_{0}\right)^{(p)}$. If $p<q$, we also have $\left(\sigma_{q} M\right)^{(p+1)} \simeq$ $\left(M_{0}\right)^{(p)}$. Thus $\sigma_{q} M$ and $\sigma_{p} M$ have the same $p$-type. The lemma follows readily.

\footnotetext{
${ }^{1}$ This is called the $(p+1)$-type in [15].
} 
Theorem 5.2. Given $p \geq 1$ and $n \geq 2 p+3$, there are homology $n$-spheres $\Sigma_{1}^{n}$ and $\Sigma_{2}^{n}$ of the same p-type, but with $\pi_{p+1}\left(\Sigma_{1}\right) ¥ \pi_{p+1}\left(\Sigma_{2}\right)$ as abelian groups.

Proof. Let $\Sigma^{3}=\Sigma(2,3,5)=S^{3} / \pi$ be the Poincare homology sphere, with $\pi=\pi_{1} \Sigma$ the binary icosahedral group of order 120. Define

$$
\Sigma_{1}^{n}=\sigma_{n-3} \Sigma^{3}, \quad \Sigma_{2}^{n}=\sigma_{n-p-3} \sigma_{p} \Sigma^{3} .
$$

By the above lemma, $\Sigma_{1}$ and $\Sigma_{2}$ have the same $p$-type. By Theorem 1.4, their universal covers are

$$
\begin{aligned}
& \widetilde{\Sigma}_{1}=\#_{1}^{119} S^{n-2} \times S^{2}, \\
& \widetilde{\Sigma}_{2}=\#_{1}^{119}\left(S^{n-2} \times S^{2} \# S^{n-p-1} \times S^{p+1} \# S^{n-p-2} \times S^{p+2}\right) .
\end{aligned}
$$

As $p+1<n-p-1 \leq n-2$, we have

$$
\begin{aligned}
& \pi_{p+1}\left(\Sigma_{1}\right) \cong \pi_{p+1}\left(\bigvee_{1}^{119}\left(S^{n-2} \vee S^{2}\right)\right) \cong \bigoplus_{1}^{119} \pi_{p+1}\left(S^{2}\right) \\
& \pi_{p+1}\left(\Sigma_{2}\right) \cong \bigoplus_{1}^{119}\left(\pi_{p+1}\left(S^{2}\right) \oplus \pi_{p+1}\left(S^{p+1}\right) \oplus \pi_{p+1}\left(S^{n-p-2}\right)\right) .
\end{aligned}
$$

Therefore

$$
\pi_{p+1}\left(\Sigma_{2}\right) \cong \begin{cases}\pi_{p+1}\left(\Sigma_{1}\right) \oplus \mathbb{Z}^{238}, & \text { if } n=2 p+3 \\ \pi_{p+1}\left(\Sigma_{1}\right) \oplus \mathbb{Z}^{119}, & \text { if } n>2 p+3\end{cases}
$$

In particular, for $n \geq 5$ we get two homology $n$-spheres with isomorphic $\pi_{1}$ but distinct $\pi_{2}$. In fact, we may get $n-3$ such manifolds by setting $\Sigma_{i}^{n}=\sigma_{n-i-2} \sigma_{1}^{i-1} \Sigma^{3}, i=1, \ldots, n-3$. Then

$$
\pi_{2}\left(\Sigma_{i}^{n}\right) \cong \begin{cases}\mathbb{Z}^{119 i}, & \text { if } i<n-3 \\ \mathbb{Z}^{119(i+1)}, & \text { if } i=n-3\end{cases}
$$

The next theorem provides examples with different $\mathbb{Z} \pi$-module structure on $\pi_{2}$. Whether there are homology 4-spheres with distinct $\pi_{2}$ (as groups or as $\mathbb{Z} \pi$-modules) is still an open question (see $[12])$.

Theorem 5.3. Given $n \geq 5$, there are homology $n$-spheres $\Sigma_{1}^{n}$ and $\Sigma_{2}^{n}$ with $\pi_{1} \Sigma_{1} \cong \pi_{1} \Sigma_{2}, \pi_{2} \Sigma_{1} \cong \pi_{2} \Sigma_{2}$ as abelian groups, but $\pi_{2} \Sigma_{1} ¥ \pi_{2} \Sigma_{2}$ as $\mathbf{Z} \pi_{1}$ modules.

Proof. Start with $\Sigma^{3}=\Sigma(2,3,7)=\mathbb{R}^{3} / \pi$-an aspherical Brieskorn homology sphere - and define $\Sigma_{1}^{n}, \Sigma_{2}^{n}$ as in Theorem 5.2. By Lemma 3.3 and the Hurewicz isomorphism we have

$$
\pi_{2} \Sigma_{1} \cong \mathbb{Z} \pi, \quad \pi_{2} \Sigma_{2} \cong \begin{cases}\mathbf{Z} \pi \oplus(I \pi)^{2}, & \text { if } n=5, \\ \mathbb{Z} \pi \oplus I \pi, & \text { if } n>5 .\end{cases}
$$

These are both free abelian groups of countable rank, but, by the proof of Corollary 3.5, are distinct as $\mathbf{Z} \pi$-modules. 
We conclude this section with examples of homology $n$-spheres distinguished by their $k$-invariants, generalizing those of [11] and [12]. Recall that the $k$ invariant of a CW-complex $K$ is a cohomology class $k(K) \in H^{3}\left(\pi_{1} K, \pi_{2} K\right)$. It behaves nicely under $p$-spinning. Indeed, for $n \geq 3$ the inclusion map $M_{0}^{n} \rightarrow \sigma_{p} M^{n}$ induces $\pi_{1} M_{0}=\pi_{1} \sigma_{p} M$ and $\pi_{2} M_{0} \subset \pi_{2} \sigma_{p} M=\pi_{2} M_{0} \oplus I \pi$, if $p=1$ and $\pi_{2} M_{0}=\pi_{2} \sigma_{p} M$, if $p>1$. Since continuous maps preserve $k$-invariants, it follows that

$$
k\left(\sigma_{p} M\right)= \begin{cases}\left(k\left(M_{0}\right), 0\right), & \text { if } p=1, \\ k\left(M_{0}\right), & \text { if } p>1 .\end{cases}
$$

Notice also that for $n \geq 4, \pi_{2} M_{0}=\pi_{2} M$ and so $k\left(M_{0}\right)=k(M)$.

Theorem 5.4. For $n \geq 3$ and $N \geq 2$, there exist $N$ homology $n$-spheres with isomorphic $\pi_{1}$ and $\pi_{2}$ (as $\mathbb{Z} \pi_{1}$-modules), but with distinct $k$-invariants.

Proof. We will construct homology $n$-spheres $\Sigma_{1}^{n}, \ldots, \Sigma_{N}^{n}$ satisfying a stronger condition. Namely, for $i \neq j$, given any isomorphism $\alpha: \pi_{1} \Sigma_{i}^{n} \rightarrow \pi_{1} \Sigma_{j}^{n}$ and any $\alpha$-homomorphism $\beta: \pi_{2} \Sigma_{i}^{n} \rightarrow \pi_{2} \Sigma_{j}^{n}$, we have $\alpha^{*}\left(k\left(\Sigma_{j}^{n}\right)\right) \neq \beta_{*}\left(k\left(\Sigma_{i}^{n}\right)\right)$.

The existence of homology $n$-spheres $\Sigma_{i}^{n}$ verifying the above condition is established for $n=3$ in [11] and for $n=4$ in [12]. For $n \geq 5$, set

$$
\Sigma_{i}^{n}=\sigma_{n-4} \Sigma_{i}^{4} \text {. }
$$

We claim $\Sigma_{i}^{n}$ satisfy the above condition.

If $n=5$, suppose there exist, for $i \neq j$, an isomorphism $\alpha: \pi_{1} \Sigma_{i}^{5} \cong \pi_{1} \Sigma_{i}^{4} \rightarrow$ $\pi_{1} \Sigma_{j}^{5} \cong \pi_{1} \Sigma_{j}^{4}$ and an $\alpha$-homomorphism $\beta: \pi_{2} \Sigma_{i}^{5} \cong \pi_{2} \Sigma_{i}^{4} \oplus I \pi \rightarrow \pi_{2} \Sigma_{j}^{5} \cong \pi_{2} \Sigma_{j}^{4} \oplus$ $I \pi$ such that $\alpha^{*}\left(k\left(\Sigma_{j}^{5}\right)\right)=\beta_{*}\left(k\left(\Sigma_{i}^{5}\right)\right)$. Let $\beta^{\prime}$ be the restriction of $\beta$ to $\pi_{2}\left(\Sigma_{i}^{4}\right)$. As $k\left(\Sigma_{i}^{5}\right)=\left(k\left(\Sigma_{i}^{4}\right), 0\right)$, we see that $\beta^{\prime}$ is an $\alpha$-homomorphism (not necessarily an isomorphism, even if $\beta$ is ) satisfying $\alpha^{*}\left(k\left(\Sigma_{j}^{4}\right)\right)=\beta_{*}^{\prime}\left(k\left(\Sigma_{i}^{4}\right)\right)$. This is a contradiction.

If $n>5$, then $\pi_{2} \Sigma_{i}^{n}=\pi_{2} \Sigma_{i}^{4}$ and $k\left(\Sigma_{i}^{n}\right)=k\left(\Sigma_{i}^{4}\right)$, so the proof is immediate.

Remark. One cannot get these high-dimensional homology spheres by directly spinning the three-dimensional ones. Indeed, for every $i$ and $j, \sigma_{p}\left(\Sigma_{i}^{3}\right)$ is diffeomorphic to $\sigma_{p}\left(\Sigma_{j}^{3}\right)$. This is why we first had to construct $\Sigma_{i}^{4}$ before being able to continue spinning.

\section{ITERATED SPUN KNOTS}

Let $K=\left(S^{n}, S^{n-2}\right), n \geq 3$, be a smooth $n$-knot. The $p$-spin of $K$ is the $n+$ $p$-knot $\sigma_{p} K=\left(\sigma_{p} S^{n}, \sigma_{p} S^{n-2}\right)$. Cappell [3] shows that if $X(K)=S^{n} \backslash S^{n-2} \times D^{2}$ is the exterior of $K$, then $X\left(\sigma_{p} K\right)=S^{p} \times X(K) \cup\left(D^{p+1} \times S^{n-2} \backslash D^{p-n-1}\right) \times S^{1}$, identified along $S^{p} \times S^{n-2} \times S^{1}$. Moreover, if $K$ is a standardly fibered knot, with fiber $F_{0}^{n-1}$, then $\sigma_{p} K$ is also standardly fibered, with fiber $\left(\sigma_{p} F\right)_{0}$. He then notices that in general $\sigma_{p+q} K \not \sigma_{q} \sigma_{p} K$. For $P$ a tuple, denote by $\sigma_{P} K$ the iterated $P$-spin of $K$. We then venture the following conjecture. 
Conjecture. If $K$ is a nontrivial knot and $P \neq Q$, then $\sigma_{P} K \not \sigma_{Q} K$.

One approach to this problem is to study the $\mathbb{Z} \pi_{1} X$-module structure of the homology groups of $\left.X \widetilde{\left(\sigma_{P}\right.} K\right)$ (of course $H_{*}\left(X\left(\sigma_{P} K\right)\right) \cong H_{*}\left(S^{1}\right)$ no longer provides any information). These groups can be computed using the description of $H_{*}\left(X\left(\widetilde{\sigma_{p} K}\right)\right)$ in [6] (see also $\left.[1,5,14]\right)$, which is similar to that of $H_{*}\left(\widetilde{\sigma_{p} M}\right)$ in Lemma 3.1. The ensuing difficulties though are much the same as for closed manifolds. We thus will content ourselves with providing some evidence for the validity of the conjecture in case the knot is fibered.

First note that if $K$ and $K^{\prime}$ are standardly fibered knots, with fibers $F_{0}$ and $F_{0}^{\prime}$, then $K \cong K^{\prime}$ implies $F \simeq F^{\prime}$. For if $Y(K)=X(K) \cup_{S^{n-2} \times S^{1}} D^{n-1} \times S^{1}$, then the universal abelian cover of $Y$ is homotopy equivalent to $F$. It now follows from Theorem 2.3 that:

If $K$ is a standardly fibered knot, with closed fiber not a homology sphere, then the conjecture is true.

In particular, if $K=\left(S^{3}, S^{1}\right)$ is fibered and nontrivial, then $P \neq Q$ implies $\sigma_{P} K \not \sigma_{Q} K$. If $K$ is a topologically nontrivial knot in $S^{4}$, its closed fiber $F^{3}$ cannot be a homotopy $S^{3}$ by the work of Freedman. It follows from Theorem 4.1 that:

If $K=\left(S^{4}, S^{2}\right)$ is a standardly fibered knot not homeomorphic to the trivial knot, then the conjecture is true.

Acknowledgment. I wish to thank R. Daverman for raising some questions which led to the results in $\S 5$.

\section{REFERENCES}

1. J. J. Andrews and D. W. Sumners, On higher-dimensional fibered knots, Trans. Amer. Math. Soc. 153 (1971), 415-426.

2. E. Artin, Zur Isotopie zweidimensionaler Flächen in $R_{4}$, Abh. Math. Sem. Univ. Hamburg, 4 (1926), 174-177.

3. S. E. Cappell, Superspinning and knot complements, Topology of Manifolds (Proc. Inst. Univ. of Georgia, Athens, 1969), Markham, Chicago, Il., 1970, pp. 358-383.

4. __ A splitting theorem for manifolds, Invent. Math. 33 (1976), 69-170.

5. D. B. A. Epstein, Linking spheres, Proc. Cambridge Philos. Soc. 56 (1960), 215-219.

6. C. Mc A. Gordon, Some higher-dimensional knots with the same homotopy groups, Quart. J. Math. Oxford 24 (1973), 411-422.

7. __ A note on spun knots, Proc. Amer. Math. Soc. 58 (1976), 361-362.

8. J. Hempel, 3-manifolds, Princeton Univ. Press, Princeton, N.J., 1976.

9. I. Kaplansky, Fields and rings, Chicago Lectures in Mathematics, Chicago Univ. Press, Chicago, Il., 1969.

10. S. P. Plotnick, Equivariant intersection forms, knots in $S^{4}$, and rotations in 2-spheres, Trans. Amer. Math. Soc. 296 (1986), 543-575.

11. S. P. Plotnick and A. I. Suciu, k-invariants of knotted 2-spheres, Comment. Math. Helv. 60 (1985), 54-84. 
12. A. I. Suciu, Homology 4-spheres with distinct k-invariants, Topology Appl. 25 (1987), 103110.

13. __ The oriented homotopy type of spun 3-manifolds, Pacific J. Math. 131 (1988), 393399.

14. D. W. Sumners, On an unlinking theorem, Proc. Cambridge Philos. Soc. 71 (1972), 1-4.

15. J. H. C. Whitehead, Combinatorial homotopy. I, Bull. Amer. Math. Soc. 55 (1949), 213-245.

Department of Mathematics, Northeastern University, Boston, Massachusetts 02115 